\title{
Nonlinear short-wave propagation in ferrites
}

\author{
R. A. Kraenkel, ${ }^{1}$ M. A. Manna, ${ }^{2}$ and V. Merle ${ }^{2}$ \\ ${ }^{1}$ Instituto de Física Teórica, Universidade Estadual Paulista, Rua Pamplona 145, 01505-900 São Paulo, Brazil \\ ${ }^{2}$ Physique Mathématique et Théorique, Université Montpellier II, CNRS -UMR 825, 34095 Montpellier, France
}

(Received 6 May 1999)

\begin{abstract}
In this paper we discuss the propagation of nonlinear electromagnetic short waves in ferromagnetic insulators. We show that such propagation is perpendicular to an externally applied field. In the nonlinear regime we determine various possible propagation patterns: an isolated pulse, a modulated sinusoidal wave, and an asymptotic two-peak wave. The mathematical structure underlying the existence of these solutions is that of the integrable sine-Gordon equation.
\end{abstract}

PACS number(s): 41.20.Jb, 02.30.Jr

The subject of this paper is the propagation of electromagnetic waves in ferrites. For our purposes, by ferrite we mean a ferromagnet of zero conductivity, a ferromagnetic insulator. We will always assume that it is in the presence of an external magnetic field and that it is saturated. In this case, due to the absence of eddy currents, electromagnetic waves may propagate. This is interesting not only from a theoretical point of view but also from a practical point of view, particularly in connection with the behavior of ferrite devices such as ferrite-loaded waveguides at microwave frequencies [1].

The equations that describe this wave propagation are fundamentally nonlinear. It is a simple exercise to show that, in the absence of currents and charges, the Maxwell equations in a medium of scalar permittivity $\tilde{\varepsilon}$ reduce to

$$
-\boldsymbol{\nabla}(\boldsymbol{\nabla} \cdot \mathbf{H})+\nabla^{2} \mathbf{H}=\frac{1}{c^{2}} \frac{\partial^{2}}{\partial t^{2}}(\mathbf{H}+\mathbf{M}),
$$

where $c=1 / \sqrt{\mu_{0} \widetilde{\varepsilon}}$ is the speed of light, $\mu_{0}$ is the magnetic permeability of the vacuum, and $\mathbf{M}$ and $\mathbf{H}$ are, respectively, the magnetization density and the magnetic induction. En passant, we have assumed the usual constitutive relations $\mathbf{D}=\tilde{\varepsilon} \mathbf{E}$ and $\mathbf{B}=\mu_{0}(\mathbf{H}+\mathbf{M})$, where the symbols have their usual textbook meaning.

Equation (1) must be supplemented with a relation between $\mathbf{M}$ and $\mathbf{H}$. This is the torque equation, which reads, under the hypothesis of zero damping,

$$
\frac{\partial \mathbf{M}}{\partial t}=-\mu_{0} \gamma \mathbf{M} \times \mathbf{H},
$$

where $\gamma$ is the gyromagnétic ratio. It is by means of this equation that nonlinearity sets in.

For sufficiently small amplitudes a linear theory can, and has, been developed $[2,3]$. We shall return to the essentials of the linear analysis later. For the moment let us notice that a fully nonlinear theory has not been developed so far, as Eqs. (1) and (2) are not exactly solvable. In order to obtain results valid in nonlinear regimes, at least weakly nonlinear, one has to resort to intermediate models, much in the same way one proceeds in hydrodynamics, where effective long-wave or short-wave models are obtained from underlying, fully non- linear, equations. These intermediate models usually introduce a novel perturbative parameter, most often the longness or shortness of the wave, and then consider particular relations between this parameter and the amplitude scale [4]. In the present case, Nakata [5] carried out a study of the nonlinear case investigating the propagation of small-amplitude long-wavelength electromagnetic waves in a saturated ferrite in the presence of an external magnetic field. Further studies where concerned with wave modulation $[6,7]$ and dissipation [8]. The propagation of long electromagnetic waves in an isotropic damped ferromagnet with free charges has also been the object of recent studies $[9,10]$.

As we stated above, these results refer to intermediate models where a long-wave approximation is supposed. This turns out, however, not to be the most interesting physical limit. The main practical interest of ferrites is that they propagate microwaves. Thus, an opposite limit, a shortwavelength limit, comes naturally into consideration. The point is this: is it possible to construct a short-wave (SW), nonlinear limit of Eqs. (1) and (2)? If it is, do the nonlinear effects show something new? We hold in this paper that the answers for these questions are positive ones.

In basic physical systems asymptotic (nonlinear) SW dynamics cannot always be isolated owing to the particularly restrictive conditions required from the associated linear dispersion relations. Hence only few results exist $[11,12]$. To our knowledge the case study here is the first one in which a complete description of SW dynamics is given from basic physical equations.

We will show that SW's propagate in a saturated ferrite only in the direction perpendicular to the external saturating magnetic field. This comes as an existence condition for SW. Granted it is satisfied, the nonlinear dynamics obeys an integrable system of nonlinear evolution equations which, for instance, can be reduced to the sine-Gordon equation. As a consequence, soliton excitations are possible, and the dynamics can be solved in this particular limit.

We shall proceed in three steps. First, we will reproduce a part of the linear analysis of [3], and study its short-wave limit. Next, we will construct a SW nonlinear model, through the standard multiple scales method, based on the results of the preceeding step. Finally, we will discuss the equations so obtained from a physical as well as a mathematical point of view. We will be able to show explicit propagating nonlinear patterns. 
In order to clear the notation, let us rescale the variables as

$$
\mathbf{M} \rightarrow \frac{\mu_{o} \gamma}{c} \mathbf{M}, \quad \mathbf{H} \rightarrow \frac{\mu_{o} \gamma}{c} \mathbf{H}, \quad \text { and } \quad t \rightarrow c t .
$$

This gives us

$$
\begin{gathered}
-\boldsymbol{\nabla}(\boldsymbol{\nabla} \cdot \mathbf{H})+\nabla^{2} \mathbf{H}=\frac{1}{c^{2}} \frac{\partial^{2}}{\partial t^{2}}(\mathbf{H}+\mathbf{M}), \\
\frac{\partial \mathbf{M}}{\partial t}=-\mu_{0} \gamma \mathbf{M} \times \mathbf{H} .
\end{gathered}
$$

To study the linear limit regime we must look at small perturbations of a given solution. We choose the uniform state $\mathbf{M}_{\mathbf{0}}=\left(M_{0} \cos \varphi, M_{0} \sin \varphi, 0\right)$ and $\mathbf{H}_{\mathbf{0}}$ $=\left(H_{0} \cos \varphi, H_{0} \sin \varphi, 0\right)$, where $M_{0}$ and $H_{0}$ are positive constants, with $H_{0}=\alpha M_{0}(\alpha>0)$ and $0<\varphi \leqslant \pi / 2$. We linearize Eqs. (4) and (5) around this state and assume for the perturbations a plane wave solution propagating along the $x$ direction, so that $\mathbf{M}=\mathbf{M}_{0}+\mathbf{m} e^{i(k x-\omega t)}$ and $\mathbf{H}=\mathbf{H}_{\mathbf{0}}+\mathbf{h} e^{i(k x-\omega t)}$, where $\mathbf{m}$ and $\mathbf{h}$ are real vectors of components $\left(m_{x}, m_{y}, m_{z}\right)$ and $\left(h_{x}, h_{y}, h_{z}\right)$, and where $k$ and $\omega$ are, respectively, the wave number and the frequency of the wave. This leads to the following dispersion relation:

$$
\begin{aligned}
M_{0}^{2}\left[\omega^{2}(1+\alpha)-\alpha k^{2}\right]\left[\omega^{2}(1+\alpha)-k^{2}\left(\alpha+\sin ^{2} \varphi\right)\right] \\
-\omega^{2}\left(\omega^{2}-k^{2}\right)^{2}=0 .
\end{aligned}
$$

The above results can be found in [3]. We will be interested in studying the short-wave limit $k \rightarrow \infty$. To do so, suppose that $k \sim \varepsilon^{-1}$, with $\varepsilon \ll 1$, and furthermore, that $\omega(k)$ is bounded or has a pole of order one in $k$, that is to say that we may write

$$
\omega=\frac{\omega_{-1}}{\varepsilon}+\varepsilon \omega_{1}+\varepsilon^{3} \omega_{3}+\cdots
$$

This assumption corresponds just to the requirement that short-waves exist in the linear limit, as with such a dispersion relation we have that the phase velocity $\omega(k) / k$ and the group velocity $\partial \omega / \partial k$ are always bounded in the short-wave limit, resulting in finite velocity propagation of geometrical characteristics and of energy. Note, furthermore, that this expansion selects one of the branches of the dispersion relation. A more detailed treatment [3] shows that it is the socalled ordinary branch, with waves propagating with velocity $c$, that is chosen. Introducing such an expression in (6) we get, at the first order in $\varepsilon$,

$$
\omega_{-1}^{2}=k_{0}^{2}
$$

In the following we will consider $\omega_{-1}=k_{0}$, hence choosing a direction of propagation. The next order leads to

$$
\sin ^{2}(\varphi)=1
$$

The meaning of this result is that a SW can propagate only if $\varphi=\pi / 2$. Condition (9) is thus an existence condition for a SW. In what follows we need also to know $\omega_{1}$, which is given by

$$
\omega_{1}=-M_{0}^{2} \frac{(1+\alpha)}{2 k_{0}} .
$$

Having put in focus the short-wave characteristics in the linear limit, we now turn to the nonlinear aspects, which constitute the main results of this paper. We will resort to the multiple-scale method, adapted to short-wave asymptotics. Thus, we will introduce rescaled, space and time variables, such that

$$
\frac{\partial}{\partial x}=\frac{1}{\varepsilon} \frac{\partial}{\partial \zeta} \quad \text { and } \quad \frac{\partial}{\partial t}=-\frac{1}{\varepsilon} \frac{\partial}{\partial \zeta}+\varepsilon \frac{\partial}{\partial \tau} .
$$

We will now consider general expansions of the form:

$$
\begin{gathered}
\mathbf{M}=\mathbf{M}^{(0)}+\varepsilon \mathbf{M}^{(1)}+\varepsilon^{2} \mathbf{M}^{(2)}+\cdots, \\
\mathbf{H}=\mathbf{H}^{(0)}+\varepsilon \mathbf{H}^{(1)}+\varepsilon^{2} \mathbf{H}^{(2)}+\cdots,
\end{gathered}
$$

and we consider that all the functions depend on the variables $\zeta$ and $\tau$. Before substituting these expansions into Eqs. (4) and (5), let us state the boundary conditions, which are $\mathbf{M}^{(0)} \rightarrow\left(0, M_{0}, 0\right), \quad \mathbf{H}^{(0)} \rightarrow\left(0, H_{0}, 0\right), \quad \mathbf{M}^{(i)} \rightarrow(0,0,0)$, and $\mathbf{H}^{(i)} \rightarrow(0,0,0)$ for $\zeta \rightarrow-\infty$. Moreover, we impose that all derivatives of $H_{i}$ and $M_{i}$ go to zero for $\zeta \rightarrow-\infty$. Using the new variables, the derivative operators, the expansion for $\mathbf{M}$ and $\mathbf{H}$ and using the new boundary values we find, at the first orders in $\varepsilon$, that $\mathbf{M}^{(0)}$ is the constant vector $\mathbf{M}^{(0)}$ $=\left(0, M_{0}, 0\right)$ and that $H_{x}^{(0)}=0$. The second order equations lead to $M_{y}^{(1)}=M_{z}^{(1)}=0$ and to the relations

$$
M_{x}^{(1)}=-H_{x}^{(1)} \quad \text { and } \quad \frac{\partial}{\partial \zeta} M_{x}^{(1)}=M_{0} H_{z}^{(0)} .
$$

The third order leads to a system of six equations which reduces, using the precedent orders, to a system of two coupled equations for the functions $H_{y}^{(0)}$ and $M_{x}^{(1)}$,

$$
\begin{gathered}
\frac{\partial}{\partial \tau} H_{y}^{(0)}=\frac{-1}{2 M_{0}} M_{x}^{(1)} \frac{\partial}{\partial \zeta} M_{x}^{(1)}, \\
\frac{\partial^{2}}{\partial \zeta \partial \tau} M_{x}^{(1)}=\frac{M_{0}}{2} M_{x}^{(1)}\left(H_{y}^{(0)}+M_{0}\right) .
\end{gathered}
$$

The above system of equations (13) and (14) is a nonlinear system describing the evolution of the first nontrivial terms in the expansion of $\mathbf{H}$ and $\mathbf{M}$. It is worth pointing out that the phenomena so described, and which will be discussed below, involve effects of $\mathcal{O}(1)$ in $\mathbf{H}$ and of $\mathcal{O}(\varepsilon)$ in M. We will now discuss the consequences of these results. These will follow from the fact that Eqs. (13) and (14) can be reduced to an integrable system, which displays exact solutions. We would like to point out that the importance of the integrability of this system comes not only from the fact that we can find exact solutions, but also, and overall, from the fact that the large time asymptotics of such systems is well determined. Indeed, the solutions for large times (or the in- 
termediate asymptotics [15]) can be qualitatively determined from the initial conditions. Thus the general picture emerges where for large enough times the solutions are composed of a train of solitons plus some radiative part.

The system of equations (13) and (14) is a $(1+1)$ dimensional reduction of a $(2+1)$-dimensional integrable system found in [13]. But instead of proceeding through dimensional reduction, we will prefer to present a straightforward transformation from the system in question to the sineGordon equation [14], and then map some well-known solutions of this equation to solutions of the system (13) and (14).

The transformation referred above is best understood if made in two steps. First, we define

$$
\begin{gathered}
M_{x}^{(1)}= \pm \Theta, \\
H_{y}^{(0)}=-M_{0}+\frac{4}{M_{0}} \eta_{\zeta},
\end{gathered}
$$

which leads us to the system

$$
\begin{gathered}
\frac{\partial^{2} \eta}{\partial \zeta \partial \tau}=-2 \Theta \frac{\partial \Theta}{\partial \zeta}, \\
\frac{\partial^{2} \Theta}{\partial \zeta \partial \tau}=2 \Theta \frac{\partial \eta}{\partial \zeta},
\end{gathered}
$$

which, through the following transformation:

$$
\begin{aligned}
& \frac{\partial \eta}{\partial \zeta}=u \cos (\mathrm{v}), \\
& \frac{\partial \Theta}{\partial \zeta}=u \sin (\mathrm{v}),
\end{aligned}
$$

takes us to the system

$$
\begin{gathered}
\frac{\partial u}{\partial \tau}=0, \\
\frac{\partial^{2} \mathbf{v}}{\partial \tau \partial \zeta}=2 u \sin (\mathbf{v}) .
\end{gathered}
$$

The corresponding boundary conditions are

$$
\begin{gathered}
\lim _{\zeta \rightarrow-\infty} u= \pm M_{0}^{2}(1+\alpha) / 4, \\
\lim _{\zeta \rightarrow-\infty} \mathrm{v}=n \pi,
\end{gathered}
$$

with $n$ an integer. It is then obvious that one solution is to take $u$ constant, $u= \pm M_{0}^{2}(1+\alpha) / 4$ and consider the evolution of $\mathrm{v}$ given by the sine-Gordon equation, which reads, after the transformation $\zeta=\xi / \sqrt{u}, \tau=\tau_{1} / \sqrt{u}$ :

$$
\frac{\partial^{2} \mathrm{v}}{\partial \xi \partial \tau_{1}}=\sin (\mathrm{v})
$$

This equation has extensively been discussed in textbooks [16]. Let us see how the solutions to Eq. (23) are reflected in

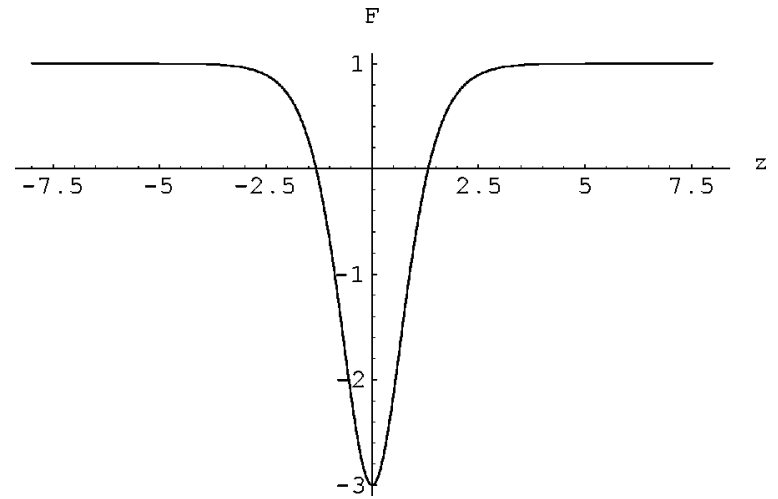

FIG. 1. The solitary-wave profile of the function $F \equiv H_{y}^{(0)}$ coming from the kink solution to Eq. (23) with $M_{0}=\alpha=1$.

the original physical variables. Here we shall treat two distinguished cases: the kink solution and the breather solution to Eq. (23).

The kink solution of the sine-Gordon equation is given by

$$
\mathbf{V}=4 \arctan [\exp (z)],
$$

with $z=k \xi+\tau_{1} / k$. We can obtain the function $H_{y}^{(0)}$ by going through the reversed transformations. We omit the details, which are purely algebraic manipulations. The result is

$$
H_{y}^{(0)}=\alpha M_{0}-2 M_{0}(1+\alpha) \operatorname{sech}^{2}(z) \equiv F(z),
$$

and is depicted in Fig 1. The result implies the possibility of propagation of short electromagnetic pulses in ferrites. In this case we can also calculate the function $M_{x}^{(1)}$, which turns out to be

$$
M_{x}^{(1)}= \pm M_{0}^{2}(1+\alpha) \operatorname{sech}(z) .
$$

The breather solutions to Eq. (23) are a family of solutions parametrized by $m$, given by:

$$
\mathbf{V}=-4 \arctan \left[\frac{m}{\sqrt{1-m^{2}}} \frac{\sin \left(\sqrt{1-m^{2}} y\right)}{\cosh (m w)}\right],
$$

where $y=\sqrt{2 u}(\zeta+\tau), \quad w=\sqrt{2 u}(\zeta-\tau), \quad$ with $\quad u=M_{0}^{2}(1$ $+\alpha) / 4$. It is possible to write down the expression for $H_{y}^{(0)}$ as

$$
H_{y}^{(0)}-\alpha M_{0}=-4 M_{0}(1+\alpha)\left[\frac{X}{1+X^{2}}\right]^{2},
$$

where

$$
X=\frac{m}{\sqrt{1-m^{2}}} \frac{\sin \left(\sqrt{1-m^{2}} y\right)}{\cosh (m w)} .
$$

This is a quite involved expression and it is worth looking at some particular cases. The first case, for $m \ll 1$, gives a modulated wave

$$
H_{y}^{(0)}-\alpha M_{0}=-4 M_{0}(1+\alpha) m^{2} \frac{\sin ^{2}[\sqrt{2 u}(\zeta+\tau)]}{\cosh ^{2}[m \sqrt{2 u}(\zeta-\tau)]} .
$$




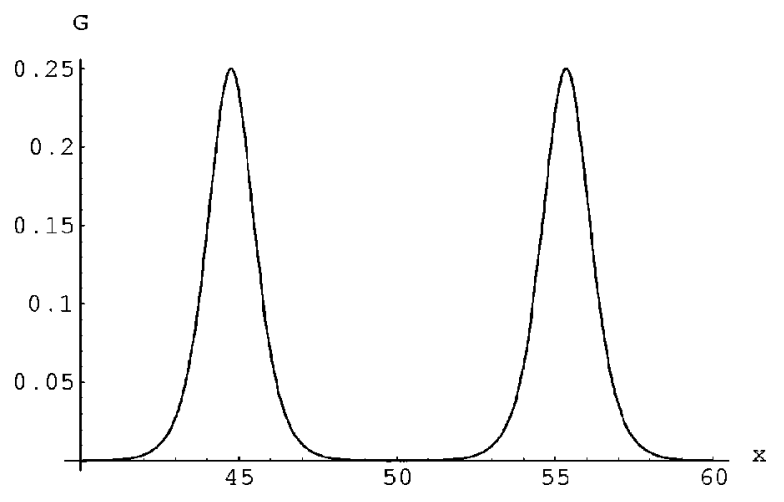

FIG. 2. Plot of $G(\zeta, \tau) \equiv\left(H_{y}^{(0)}-\alpha M_{0}\right) /\left[-4 M_{0}(1+\alpha)\right]$ given by Eq. (31) for $\tau=50$.

This represents a wave of small wavelength whose amplitude is modulated by the $\cosh ^{-1}$ term over a larger space scale. Indeed the envelope propagates in the opposite direction as the wave itself, with the same speed, and the typical wavenumber is proportional to $m \ll 1$.

Finally, from Eq. (28) in the case $m=1$ we get

$H_{y}^{(0)}-\alpha M_{0}=-4 M_{0}(1+\alpha)\left[\frac{(\zeta+\tau)^{2} \operatorname{sech}^{2}(\zeta-\tau)}{\left[1+(\zeta+\tau)^{2} \operatorname{sech}^{2}(\zeta-\tau)\right]^{2}}\right]$.
This expression does not describe a solitary wave, but its large time limits composed of two single waves of the same amplitude. In Fig. 2 we plot the function

$$
G(\zeta, \tau)=\frac{H_{y}^{(0)}-\alpha M_{0}}{-4 M_{0}(1+\alpha)}
$$

for $\tau=50$.

Let us now summarize these results. The system considered is a ferromagnetic insulator, modeled to be infinitely extended, and under the influence of an external magnetic field. Propagation of electromagnetic waves is possible under these circumstances. This propagation is essentially nonlinear. We have studied this system asymptoticaly, for short wavelengths, which we knew to exist in the linear limit. It turns out that (i) propagation can occur only perpendicular to the external field; (ii) the system of equations describing the SW limit is integrable and can be mapped to the sine-Gordon equation; (iii) propagation of pulses as well as of modulated waves are predicted. We note also that when speaking of pulses, we refer here not to sinusoidal waves but rather to propagating magnetic field configurations accompanied by a magnetization wave.

The authors thank P.G. Estévez and J. Léon for useful discussions. This work was partially funded by CAPES, FAPESP, and CNRS.
[1] R.F. Soohoo, Theory and Application of Ferrites (PrenticeHall, London, 1960).

[2] C.L. Hogan, Rev. Mod. Phys. 25, 253 (1953).

[3] A.D.M. Walker and J.F. McKenzie, Proc. R. Soc. London, Ser. A 399, 217 (1985).

[4] J. Kevorkian and J. D. Cole, Multiple Scale and Singular Perturbation Methods (Springer, New York, 1996).

[5] I. Nakata, J. Phys. Soc. Jpn. 60, 77 (1991).

[6] H. Leblond and M. Manna, J. Phys. A 27, 3245 (1994).

[7] H. Leblond and M. Manna, Phys. Rev. E 50, 2275 (1994).

[8] H. Leblond and M. Manna, J. Phys. A 26, 6451 (1993).

[9] M. Daniel, V. Veerakumar and R. Amuda, Phys. Rev. E 55,
3619 (1997).

[10] V. Veerakumar and M. Daniel, Phys. Rev. E 57, 1197 (1998).

[11] M.A. Manna and V. Merle, Phys. Rev. E 57, 6206 (1998).

[12] S.M. Gama, R. A. Kraenkel, and M.A. Manna, (unpublished).

[13] M. Boiti, J. Leon, and F. Pempinelli, Inverse Probl. 3, 371 (1987).

[14] P.G. Estévez (private communication).

[15] G.I. Barenblatt, Scaling, Self-similarity, and Intermediate Asymptotics (Cambridge University Press, Cambridge, 1996).

[16] R.K. Dodd, J.C. Eilbeck, J.D. Gibbon, and H.C. Morris, Solitons and Nonlinear Wave Equation (Academic Press, London, 1982). 